\title{
Novel syngeneic mouse mammary carcinoma cell lines from aggressive ErbB2/Neu-overexpressing/PTEN-deficient tumors
}

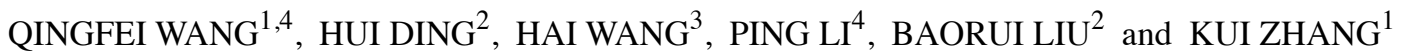 \\ ${ }^{1}$ Department of Laboratory Medicine, Nanjing Drum Tower Hospital; ${ }^{2}$ The Comprehensive Cancer Center and \\ Department of Oncology, Nanjing Drum Tower Hospital; ${ }^{3}$ Department of Pathology at Jinling Hospital, School of Medicine, \\ Nanjing University, Nanjing, Jiangsu 210008, P.R. China; ${ }^{4}$ Department of Molecular and Cellular Oncology, \\ The University of Texas MD Anderson Cancer Center, Houston, TX 77030, USA
}

Received August 13, 2014; Accepted October 13, 2014

DOI: $10.3892 /$ or.2014.3574

\begin{abstract}
Breast cancer cell lines and mouse models are valuable tools for investigating the biology of and developing potential therapeutics for human breast carcinoma. The $\mathrm{PTEN}^{-/} / \mathrm{NIC}$ mouse is a genetically engineered mouse model for ErbB2/Neu-overexpressing/-PTEN deficient breast carcinoma with histopathological and molecular features relevant to the luminal subtype of primary human breast cancer. However, the PTEN ${ }^{-/} / \mathrm{NIC}$ model develops multifocal and aggressive mammary tumors with a short life-span, which greatly impedes its preclinical usage. To complement the genetic engineering approach and to facilitate the future application of this model, in the present study, two newly established cell lines, NICP20 and NICP21, from PTEN ${ }^{-/} / \mathrm{NIC}$ mammary tumors are described. These NICP20 and NICP21 cells retained the crucial molecular phenotype similar to the origin, as confirmed by genotyping and western blot analysis. These cells induced tumors in immunocompetent syngeneic mice by mammary fat pad injection and produced lung metastasis when injected intravenously. Tumors induced by these cells displayed luminal-like histologic morphology and hyperactivation of Akt which are similar to PTEN ${ }^{-/} / \mathrm{NIC}$ tumors. Immunohistochemical staining also revealed that tumors induced by the NICP20 and NICP21 cells showed a high proliferative level, comparable angiogenesis and T-cell infiltration properties similar to $\mathrm{PTEN}^{-1} / \mathrm{NIC}$ tumors. Therefore, these NICP20 and NICP21 cells represent an alternative and useful model system to enhance our understanding of the nature of ErbB2-positive breast cancers, particularly accompanying PTEN loss and to facilitate further experimental therapeutic studies.
\end{abstract}

Correspondence to: Professor Kui Zhang or Dr Qingfei Wang, Department of Laboratory Medicine, Nanjing Drum Tower Hospital, School of Medicine, Nanjing University, 321 Zhongshan Road, Nanjing, Jiangsu 210008, P.R. China

E-mail: zkangkui@gmail.com

E-mail: wangqingfei1020@hotmail.com

Key words: breast cancer, mammary tumor, ErbB2/Neu, PTEN, syngeneic

\section{Introduction}

Breast cancer remains one of the most common malignancies and is the second leading cause of cancer-related mortality in women (1). Standard treatment modalities have improved the overall survival and the quality of life of patients. However, more personalized therapies or improvement of existing treatment are needed due to the heterogeneity of breast cancer and resistance to existing therapies. The molecular profiling of human breast cancers has identified at least 5 subtypes with distinct clinical outcomes (2-5). One of the common subgroups is the ErbB2-positive subtype, which occurs in $20-30 \%$ of breast cancer cases. It also has been demonstrated that elevated expression/amplification of ErbB2 correlates with poor prognosis (6-8).

Mutation or deficiency of the tumor-suppressor phosphatase and tensin homologue (PTEN) has been reported to occur in $5-10 \%$ of human breast cancer cases (9). Loss of PTEN function results in hyperactivation of the PI3K/AKT pathway and induction of basal-like breast cancers $(10,11)$. Furthermore, numerous studies have indicated that loss of PTEN confers resistance to trastuzumab (Herceptin), a humanized monoclonal antibody targeting ErbB2 (12-14). The above findings suggest that PTEN disruption may play a critical role in ErbB2positive human breast cancer. To directly evaluate the effect of PTEN loss on ErbB2-induced mammary tumorigenesis and progression, Shade et al generated the PTEN-deficient/NIC genetically engineered mouse model. This novel model utilized the murine mammary tumor virus (MMTV) promoter to drive co-expression of activated ErbB2/Neu and Cre recombinase coupling PTEN conditional depletion in the same mammary epithelial cells (15). PTEN-deficient/NIC mice exhibited rapid formation of highly metastatic mammary tumors and displayed histopathological and molecular features characteristic of the luminal subtype of primary human breast cancer $(15,16)$. We found previously that loss of both PTEN alleles $\left(\mathrm{PTEN}^{-/} / \mathrm{NIC}\right.$ mice) resulted in significant resistance to $\mathrm{Neu}$ antibody treatment (17). Therefore, $\mathrm{PTEN}^{-1 /} / \mathrm{NIC}$ mice represent a valuable tool for biological study and drug discovery for trastuzumabresistant ErbB2/Neu-positive breast cancer.

However, there are several disadvantages and challenges of using the PTEN ${ }^{-/} / \mathrm{NIC}$ model to preclinically evaluate novel 
therapeutics. First, it has a high cost, is time consuming and is labor intensive to obtain cohorts of female PTEN ${ }^{-1 /} / \mathrm{NIC}$ mice. Second, it may be very difficult to quantify tumor size during preclinical investigations due to the fact that the $\mathrm{PTEN}^{-/} / \mathrm{NIC}$ model develops multifocal and aggressive mammary tumors. To partially overcome the major drawbacks and complement the genetic engineering approach, we attempted to establish cell lines from spontaneous mammary tumors that arose in the $\mathrm{PTEN}^{-/} / \mathrm{NIC}$ mice. In the present study, two cell lines derived from PTEN ${ }^{-/} / \mathrm{NIC}$ mammary tumors were generated and characterized both in vitro and in vivo, providing an alternative and useful resource for future studies.

\section{Materials and methods}

Cell lines and cell culture. Cells were cultured in growth medium [DMEM/F12 with 10\% FBS, $5 \mu \mathrm{g} / \mathrm{ml}$ insulin, $10 \mathrm{ng} / \mathrm{ml}$ epidermal growthfactor(EGF), $1 \mu \mathrm{g} / \mathrm{mlhydrocortisone}, 35 \mu \mathrm{g} / \mathrm{ml}$ bovine pituitary extract and $100 \mathrm{U}$ penicillin/streptomycin]. The cell number was evaluated on a cell counter. TM15 cells were established from a spontaneous ErbB2/Neu-positive/ PTEN wild-type $\left(\mathrm{PTEN}^{+/} / \mathrm{ErbB}^{\mathrm{KI}}\right)$ mammary tumor $(18,19)$. MT104T is an immortalized ErbB2/Neu-positive/PTENdeficienct carcinoma cell line isolated from $\mathrm{PTEN}^{-/} / \mathrm{ErbB}^{\mathrm{KI}}$ mammary tumors $(17,20)$. NICP20 and NICP21 cells were established from mammary tumors of $\mathrm{PTEN}^{-/ /} \mathrm{NIC}$ genetically engineered $\mathrm{FVB} / \mathrm{N}$ female mice, following protocols essentially as described previously (21). Briefly, fresh mammary tumors from the PTEN ${ }^{-1-} / \mathrm{NIC}$ mice were excised and minced to roughly $1 \mathrm{~mm}^{3}$ pieces, and then digested in collagenase ( $2 \mathrm{mg} / \mathrm{ml}$; Sigma) containing DMEM/F12 medium. The cells were resuspended and cultured in DMEM/F12 containing $40 \%$ Matrigel(BD Biosciences). After 2 weeks, cells were dissociated by dispase (Sigma) treatment and cultured in growth medium for $24 \mathrm{~h}$. Single cells, generated by digestion with $0.05 \%$ trypsin (Invitrogen) were plated at 1,000 cells $/ 10-\mathrm{cm}$ dish. Pooled colonies were expanded. Cells were then digested and resuspended in $100 \mu \mathrm{l}$ phosphate-buffered saline (PBS)/Matrigel (1:1) for mammary fat pad injection. Eight weeks post-injection, the cells from the resulting orthotopic tumors were explanted for a second round in vitro culture. The NICP20 and NICP21 cells were derived from selected colonies that originated from two separate $\mathrm{PTEN}^{-/} / \mathrm{NIC}$ mice.

Mammary fat pad and tail vein injection of cells. Confluent cells (70-80\%) were digested with trypsin and washed with PBS. Cells were counted and resuspended to a final concentration. For mammary fat pad injection, $10^{4}, 5 \times 10^{4}, 10^{5}, 5 \times 10^{5}$ or $10^{6}$ cells in $100 \mu \mathrm{l} \mathrm{PBS} /$ Matrigel (1:1) were injected into the \#2 inguinal mammary gland of $\mathrm{FVB} / \mathrm{N}$ female mice at 8 weeks of age. For tail vein injection, mice were anesthetized and $2 \times 10^{4}$ cells in $100 \mu \mathrm{l}$ PBS were injected using a 1-ml syringe (29 G; BD insulin syringe). FVB/N mice were obtained from the Harlan Laboratory. All animal experiments were performed according to the Guidelines for the Institutional Animal Care and Use Committee of The University of Texas M.D. Anderson Cancer Center and School of Medicine, Nanjing University.

Polymerase chain reaction (PCR) for genotyping. Genomic DNA was extracted from the cells at $70-80 \%$ confluency by digestion with proteinase K (Sigma) in $200 \mu 1$ of lysis buffer in a $60^{\circ} \mathrm{C}$ incubator for $2 \mathrm{~h}$, followed by heating at $95^{\circ} \mathrm{C}$ for $10 \mathrm{~min}$ to inactivate the enzyme. After centrifugation, $1 \mu \mathrm{l}$ of the supernatant was used for PCR reaction. Primer sequences for NEU were: 5'-TTCCGGAACCCACATCAGGCC-3' and 5'-GT TTCCTGCAGCAGCCTACGC-3'; for CRE, 5'-TGCTCTGTC CGTTTGCCG-3' and 5'-ACTGTGTCCAGACCAGGC-3'.

Immunoblotting. Cells in monolayer were washed with ice cold-PBS and harvested by scraping in lysis buffer (1\% Triton X-100, $50 \mathrm{mM}$ HEPES pH 7.4, $150 \mathrm{mM} \mathrm{NaCl}, 1.5 \mathrm{mM}$ $\mathrm{MgCl}_{2}, 1 \mathrm{mM}$ EGTA, $100 \mathrm{mM} \mathrm{NaF}, 10 \mathrm{mM}$ Na pyruvate, $1 \mathrm{mM} \mathrm{Na} \mathrm{VO}_{4}, 10 \%$ glycerol) containing protease inhibitors (Sigma) and phosphatase inhibitors (Roche). The lysate was incubated on ice for $20 \mathrm{~min}$ and centrifuged at 15,000 x g for $15 \mathrm{~min}$ at $4^{\circ} \mathrm{C}$. The protein concentration of the supernatant was determined by BCA assay (Thermo Scientific), and $30 \mu \mathrm{g}$ total protein was electrophoresed on $10 \%$ SDS-PAGE and then transferred onto a nitrocellulose membrane (Bio-Rad). Pten, pAkt S473 and Akt antibodies were obtained from Cell Signaling Technology. Antibodies for ErbB2/Neu and $\beta$-actin were obtained from Santa Cruz Biotechnology and Sigma, respectively. The blots were incubated with HRP-conjugated secondary antibodies and visualized by ECL (Amersham).

Whole mount and histology. Number 4 mammary gland was excised from 4 - to 6-week old virgin female $\mathrm{PTEN}^{-/} / \mathrm{NIC}$ mice and spread on glass slides for overnight fixation in $4 \%$ paraformaldehyde. The next day the samples were hydrated and stained in a filtered solution of $0.2 \%$ carmine (Sigma) and $0.5 \%$ aluminum potassium sulfate for 1-2 days. Glands were then dehydrated sequentially through 70,90 and $100 \%$ ethanol for $15 \mathrm{~min}$ each, precleared in toluene and stored in methylsalicylate. Lung whole mount preparations were harvested and infused with formalin. Tumor tissues and lungs were fixed in formalin, processed routinely and embedded in paraffin. For histological analysis, paraffin sections $(4-\mu \mathrm{m})$ were stained with hematoxylin and eosin according to standard protocols.

Immunohistochemistry. Paraffin-embedded tumor sections $(4-\mu \mathrm{m})$ were subjected to antigen retrieval in a pressure cooker with sodium citrate buffer $(\mathrm{pH}, 6.0)$ and incubated with antibodies specific for phospho-Akt S473 (1:100; Cell Signaling), Ki-67 (1:200; Dako), CD34 (1:50; eBioscience), CD3 (1:300; Epitomics) overnight at $4^{\circ} \mathrm{C}$. Biotin-conjugated secondary antibodies were used. Remaining steps were performed using Vectastain ABC kits (Vector Laboratories). Slides were counterstained with hematoxylin. The stained slides were evaluated by two pathologists, and images were acquired using a Zeiss microscope with Axiovision software (Carl Zeiss, Inc.).

\section{Results}

PTEN $^{-/-/ N I C ~ m i c e ~ d e v e l o p ~ m u l t i f o c a l ~ a n d ~ a g g r e s s i v e ~}$ mammary tumors. Genetic structure and general procedures to generate the $\mathrm{PTEN}^{-/} / \mathrm{NIC}$ mice are depicted in Fig. 1A. $\mathrm{PTEN}^{-/} / \mathrm{NIC}$ mice developed tumors rapidly with a latency of 28 to 43 days (data not shown), which was consistent with previous results $(15,17)$. As shown in Fig. 1B, a tumor mass was found in the inguinal mammary gland from a female 

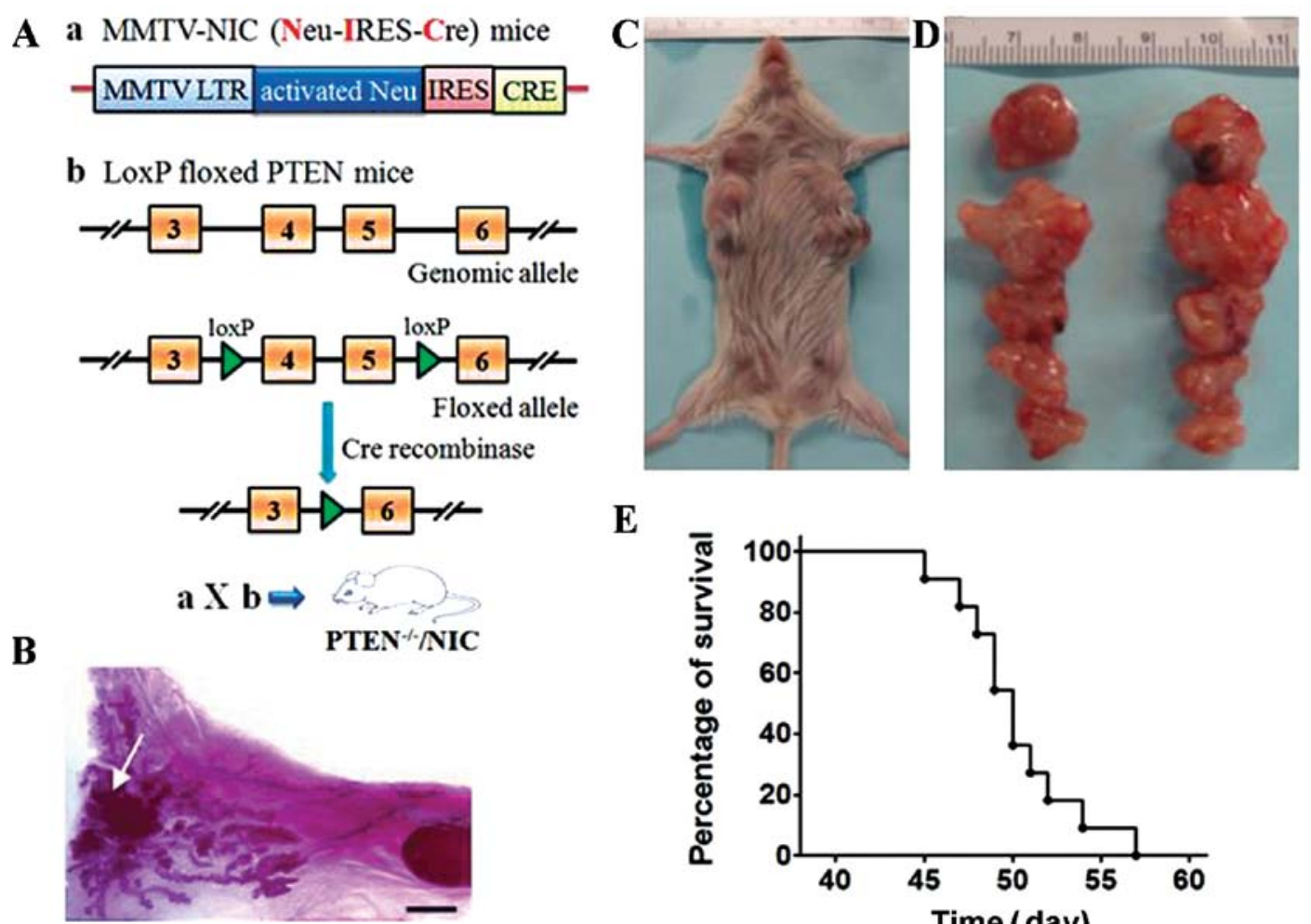

$\mathbf{E}$

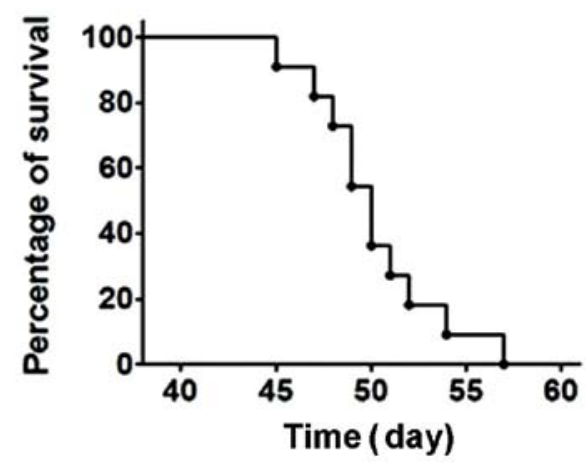

Figure 1. PTEN ${ }^{-/} / \mathrm{NIC}$ mice develop multifocal and aggressive mammary tumors. (A) Genetic structure and generation of PTEN ${ }^{-/} / \mathrm{NIC}$ mice are depicted. (B) Whole-mount staining of an inguinal mammary gland from a female PTEN ${ }^{-/} / \mathrm{NIC}$ mouse at 30 days of age. Scale bar, $1 \mathrm{~cm}$. The arrow indicates the tumor mass. (C and D) Gross observation of a female PTEN ${ }^{-/} / \mathrm{NIC}$ mouse with multifocal mammary tumors (C) and appearance of the dissected tumors (D). (E) The overall survival curve of the female $\mathrm{PTEN}^{-/-} / \mathrm{NIC}$ mice $(\mathrm{n}=11)$. Median survival time was 50 days. PTEN, phosphatase and tensin homologue.

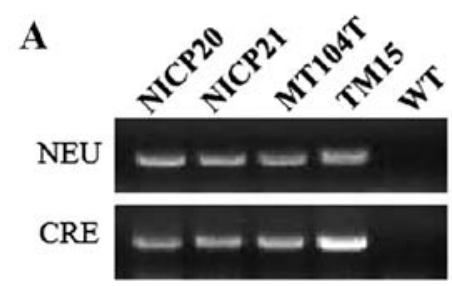

Akt

$\beta$-actin
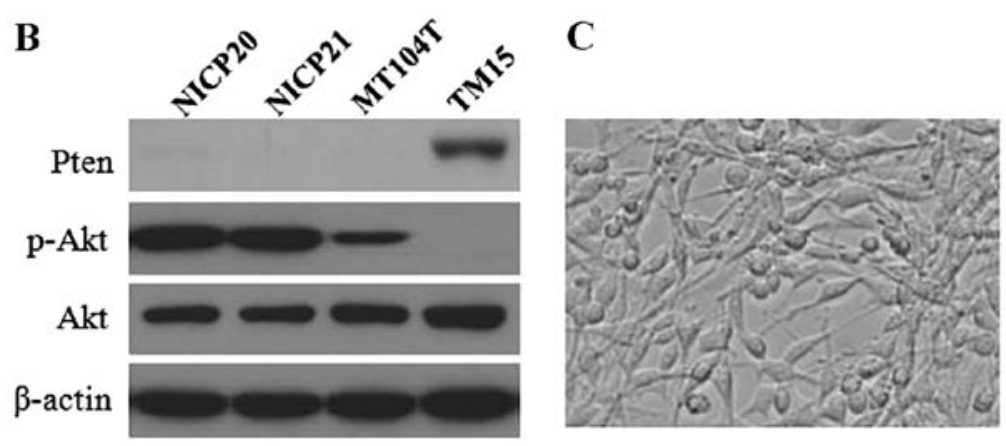

Figure 2. Establishment and characterization of the ErbB2/Neu-positive/PTEN-deficient mammary tumor cells from the PTEN ${ }^{-/} / \mathrm{NIC}_{\text {mice }}$ in vitro. (A) Genotyping for NEU and CRE using genomic DNA from the indicated cells. (B) Western blot analysis for Pten and phospho-Akt using lysates

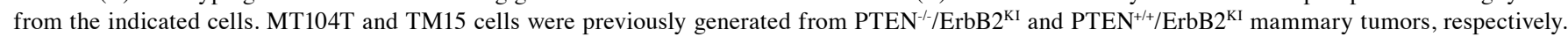
(C) Representative image of the cultured cell lines from PTEN ${ }^{-1 /} /$ NIC tumors. PTEN, phosphatase and tensin homologue.

$\mathrm{PTEN}^{-/ /}$NIC mouse at 30 -days old. All female PTEN ${ }^{-1-} / \mathrm{NIC}$ mice developed multifocal mammary tumors (Fig. 1C and D). Due to the rapid tumor growth and progression, most female $\mathrm{PTEN}^{-/ /} / \mathrm{NIC}$ mice had to be euthanized three to four weeks after initial tumor detection (tumor size $>15 \mathrm{~mm}$ in diameter). The overall survival curve of these female mice is shown in Fig. $1 \mathrm{E}$ with a median survival time of 50 days.

Establishment and characterization of the mammary tumor cells from PTEN $N^{-1} / N I C$ mice in vitro. Given the multifocal and aggressive features of the $\mathrm{PTEN}^{-/} / \mathrm{NIC}$ tumors, it was difficult to follow tumor growth. Meanwhile, to partially overcome the drawbacks of the genetic engineered model (e.g. high cost, time consuming and labor intensive), we attempted to establish cell lines from tumors that arose in the $\mathrm{PTEN}^{-1 /} / \mathrm{NIC}$ mice. According to protocols as previously described (21), we obtained two cell lines, named NICP20 and NICP21, respectively. Firstly, the NEU and CRE transgenes in the NICP20 and NICP21 cells were confirmed by PCR (Fig. 2A). As expected, western blot analysis showed no detectable PTEN expression and a high level of activated Akt both in the NICP20 and NICP21 cell lines (Fig. 2B). These results revealed that the NICP20 and NICP21 cells retained the critical molecular phenotype similar to the origin. Moreover, gross appearance 

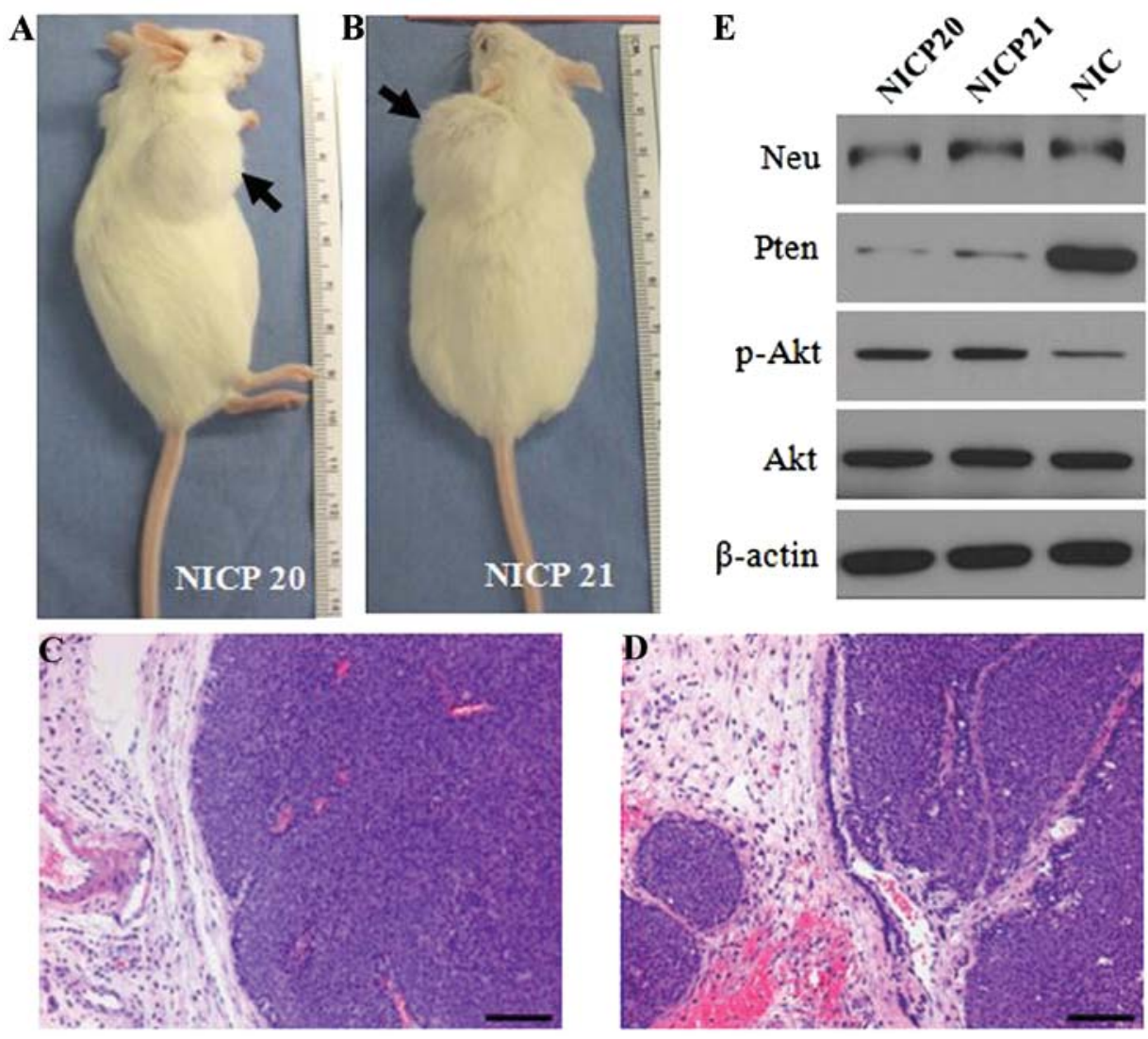

Figure 3. Tumorigenesis of the NICP20 and NICP21 cells in syngeneic mice. (A and B) Gross appearance of tumors (arrow) formed by injection of NICP20 [(A) 7 weeks post injection of $10^{5}$ cells] and NICP21 [(B) 8 weeks post injection of $5 \times 10^{4}$ cells] cells into the mammary fat pad of wild-type FVB/N female mice. (C and D) Representative hematoxylin and eosin-stained sections of tumors induced by (C) NICP20 and (D) NICP21 cells. Scale bar, $100 \mu \mathrm{m}$. (E) Western blot analysis of the indicated proteins using lysates from the tumors induced by NICP20 and NICP21 cells, respectively. Lane NIC was lysate from a spontaneous mammary tumor of an MMTV-NIC (PTEN wild-type) mouse. PTEN, phosphatase and tensin homologue.
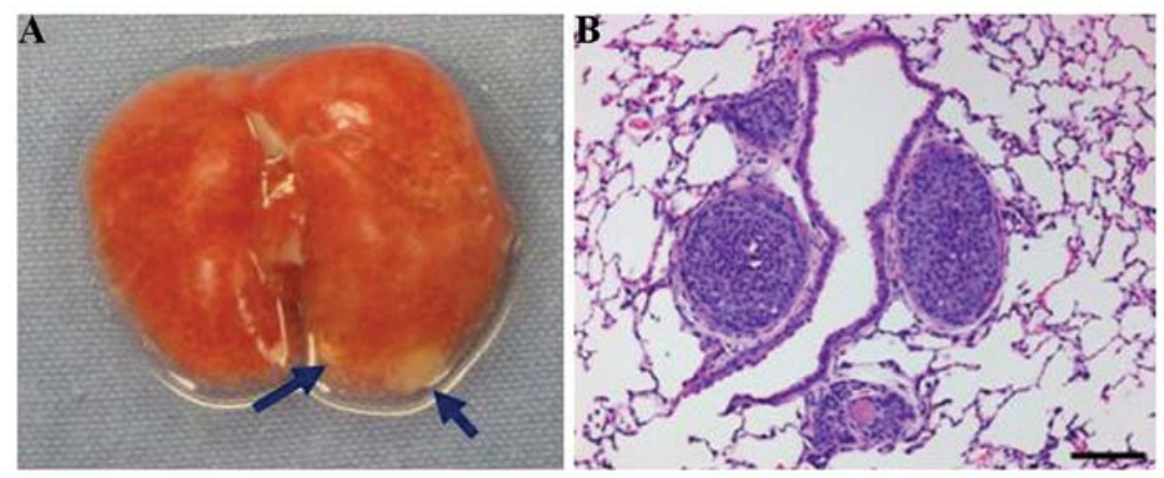

Figure 4. Lung metastasis of the established cell line in syngeneic mice by tail vein injection. (A) Macroscopic observation of lung metastasis (arrows) on day 35 post injection through the tail vein with NICP20 cells $\left(10^{4}\right)$ in a wild-type FVB/N mouse. (B) A representative hematoxylin and eosin-stained image of lung metastatic nodules by NICP20 cells under a microscope. Scale bar, $100 \mu \mathrm{m}$. PTEN, phosphatase and tensin homologue.

of the cultured cells displayed epithelial morphology over subsequent passages (Fig. 2C).

Tumorigenesis and lung metastasis of the NICP2O and NICP21 cells in syngeneic mice. After generation of the above two ErbB2/Neu-positive/PTEN-deficient cell lines, the tumorigenic ability of these cells in immune intact, syngeneic $\mathrm{FVB} / \mathrm{N}$ female mice was initially evaluated. The initial round of intramammary injections utilized $10^{6}, 5 \times 10^{5}, 10^{5}, 5 \times 10^{4}$ or $10^{4}$ cells for each cell line. By 2-5 weeks post injection, all mice had palpable tumor nodules in the mammary glands. Seven weeks post injection of $10^{5}$ NICP20 cells (Fig. 3A) and 8 weeks post injection of $5 \times 10^{4}$ NICP21 cells (Fig. 3B), the tumors were $>15 \mathrm{~mm}$ in diameter and the mice were euthanized. Histological analysis revealed that the tumors induced by the NICP20 and NICP21 cell lines had large, solid nodular nests that resembled luminal-like histologic features (Fig. 3C and D) similar to the morphology of the original $\mathrm{PTEN}^{-1 /} / \mathrm{NIC}$ tumors. Expression of ErbB2/Neu and PTEN protein in the tumors induced by NICP 20 and NICP 21 cells was also determined by 

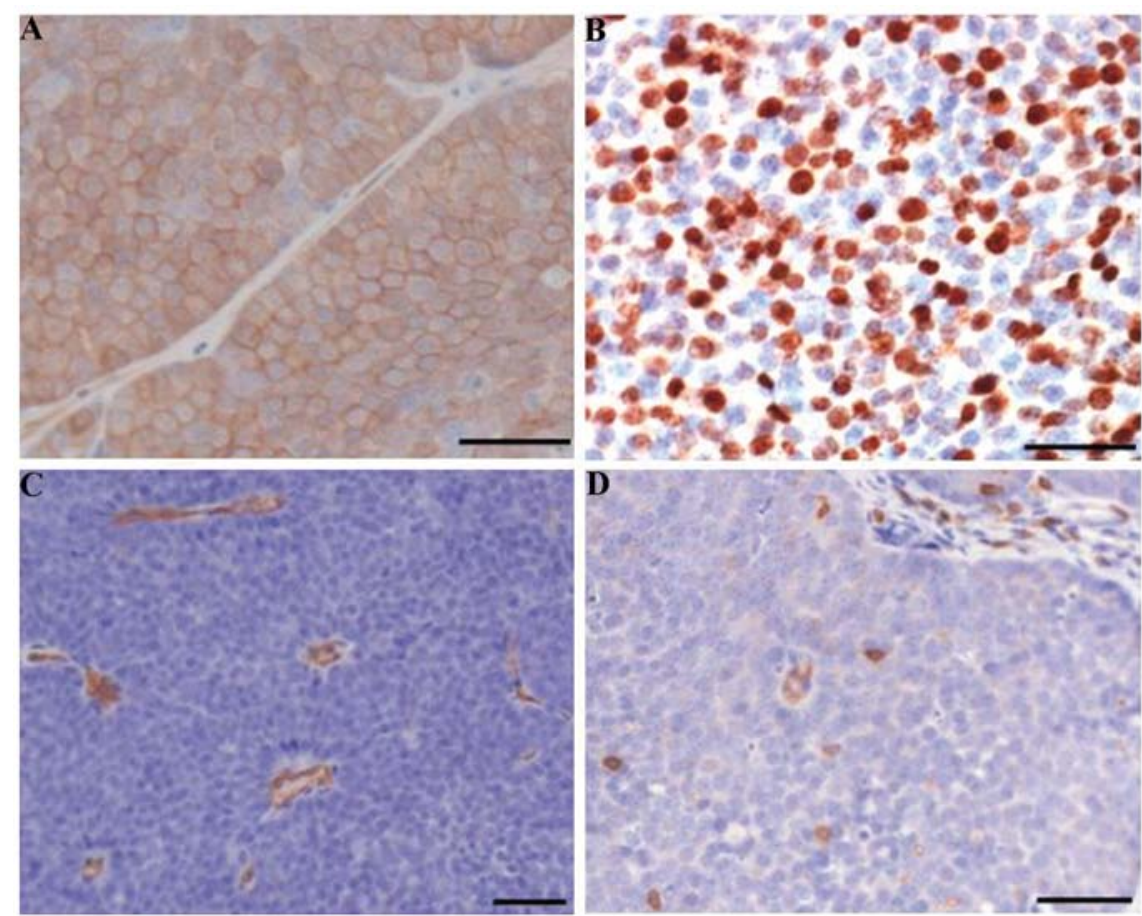

Figure 5. Immunohistochemical characterization of the orthograft tumors formed by NICP20 or NICP21 cells. (A-D) Immunohistochemistry of (A) phosphoAkt, (B) Ki67 for proliferation, (C) CD34 for blood vessel density and (D) CD3 for T cells. Scale bar, $50 \mu \mathrm{m}$. PTEN, phosphatase and tensin homologue.

western blotting (Fig. 3E). The weak bands of PTEN protein confirmed the expression of PTEN in the stromal tissue but not in the tumor cells. As expected, loss of PTEN increased Akt phosphorylation relative to the PTEN wild-type MMTV-NIC tumors (Fig. 3E). Meanwhile, the lung tissues of all the tumor-bearing mice (induced by NICP20 or NICP21 cells) were examined macroscopically and microscopically when euthanized, and no metastatic lesion was found. Base on this findings, tail vein injections were performed using different cell concentrations. Both cell lines produced lung metastasis when only $10^{4}$ cells were injected through the tail vein (Fig. 4).

Akt hyperactivation, high proliferation, angiogenesis and immune-cell infiltration of tumors formed by NICP2O and NICP 21 cells. To determine whether the in vivo characteristics of the NICP20 and NICP21 cells resemble the features of the original PTEN ${ }^{-/} / \mathrm{NIC}$ tumors, serial immunohistological analyses were further performed. Examination of phospho-Akt staining revealed that tumors formed by NICP20 or NICP21 cells displayed hyperactivation of Akt (Fig. 5A). As shown in Fig. 5B, these tumors displayed a high proliferative level. Moreover, microvessel density reflected by CD34-positive endothelium (Fig. 5C) and CD3-positive cells (Fig. 5D) per area of tumor epithelium in the cell line-induced tumors also revealed comparable angiogenesis and T-cell infiltration properties similar to the in $\mathrm{PTEN}^{-/} / \mathrm{NIC}$ tumors $(15,17)$.

\section{Discussion}

Genetically engineered mouse models of human breast cancer have contributed substantially to our understanding of the initiation and progression of breast cancer and have emerged as valuable tools for preclinical research (22-24). Breast cancer is a complex and heterogeneous disease that has distinct histopathological features, genetic variability and diverse clinical outcomes. Overexpression of the ErbB2 oncogene and loss of tumor-suppressor PTEN are both observed in certain human breast cancer cases. The FVB/N PTEN ${ }^{-/} / \mathrm{NIC}$ genetically engineered mouse was originally generated to better study the role of PTEN in ErbB2/Neu-induced mammary tumorigenesis (15). Notably, the $\mathrm{PTEN}^{-/} / \mathrm{NIC}$ model developed multifocal, highly invasive mammary tumors with histopathological and molecular features relevant to the luminal subtype of primary human breast cancer (15). Our data confirmed the aggressive features of this model with an extremely short lifespan (Fig. 1).

To facilitate our understanding and ease the future application of this well-defined model, cell lines were generated from $\mathrm{PTEN}^{-/} / \mathrm{NIC}$ mammary tumors in the present study. With the generation of the in vitro cell lines from $\mathrm{PTEN}^{-/ /} / \mathrm{NIC}$ mammary tumors and development of the syngeneic xenograft model, an additional model system for the investigation of the interplay between ErbB-2/Neu and PTEN function/signaling in mammary tumor occurrence, progression and therapies is available, possessing the advantages of easy use, troublefree monitoring of tumor growth, relatively inexpensive cost as well as the potential for in vitro manipulation. Similarly, Sahin et al reported that syngeneic transplantation of tumors from $\mathrm{PTEN}^{-/} / \mathrm{NIC}$ mice is an effective way to generate large cohorts to test therapeutics (25). Thus, the availability of our cell line model and the syngeneic transplantation approach may provide tractable alternatives for optimizing $\mathrm{PTEN}^{-/} / \mathrm{NIC}$ mice as powerful preclinical models.

Given that PTEN is a negative regulator of the PI3K/Akt pathway, its loss can lead to hyperactivation of Akt. As expected, the NICP20 and NICP21 cells had high levels of activated Akt 
in vitro (Fig. 2B) and immunohistochemical staining also localized the activated Akt on tumor cells in vivo (Fig. 5A). Hyperactivation of Akt has been associated with trastuzumab resistance and Akt has been proposed as a potential target to overcome trastuzumab resistance $(12,26-28)$. Novel inhibitors can be tested and evaluated using these NICP20 and NICP21 cells in vitro and in vivo in immunocompetent mice. In addition to inhibiting oncogenic signaling of tumor cells (29) (autonomous mechanism), studies have revealed that the immune response or stroma-tumor interactions in the tumor microenvironment (non-autonomous mechanism) are involved in the therapeutic activities of trastuzumab alone or in combination with other agents (17,30-32). Since the NICP20 and NICP21 cell-induced tumors showed comparable angiogenesis and T-cell infiltration properties as those of $\mathrm{PTEN}^{-/} / \mathrm{NIC}$ tumors (Fig. 5), they may also be useful to investigate the roles of non-autonomous mechanisms on the therapeutic response of potential treatment, by working with syngeneic wild-type or related knockout mice.

In summary, we provide an additional valuable mouse mammary carcinoma cell resource to enhance our understanding of the nature of ErbB2-positive breast cancers, particularly accompanying PTEN loss, and to facilitate experimental therapeutic studies.

\section{Acknowledgements}

We thank Dr William Muller at McGill University for kindly providing the mouse strains and the TM15 cells, and Dr Dihua Yu at the University of Texas MD Anderson Cancer Center for her full support of this study. The present study was supported by a grant from the National Natural Science Foundation of China (no. 81302241), the Key Project of Medical Science and Technology Development Foundation from Nanjing Department of Health (YKK13066), and funding from the Scientific Research Service of the Central Universities (Q.W.).

\section{References}

1. Siegel R, Naishadham D and Jemal A: Cancer statistics, 2013 CA Cancer J Clin 63: 11-30, 2013.

2. Cancer Genome Atlas Network: Comprehensive molecular portraits of human breast tumours. Nature 490: 61-70, 2012.

3. Perou CM, Sørlie T, Eisen MB, et al: Molecular portraits of human breast tumours. Nature 406: 747-752, 2000.

4. Sørlie T, Perou CM, Tibshirani R, et al: Gene expression patterns of breast carcinomas distinguish tumor subclasses with clinical implications. Proc Natl Acad Sci USA 98: 10869-10874, 2001.

5. Sotiriou $\mathrm{C}$ and Piccart MJ: Taking gene-expression profiling to the clinic: when will molecular signatures become relevant to patient care? Nat Rev Cancer 7: 545-553, 2007.

6. Slamon DJ, Clark GM, Wong SG, Levin WJ, Ullrich A and McGuire WL: Human breast cancer: correlation of relapse and survival with amplification of the HER-2/neu oncogene. Science 235: 177-182, 1987.

7. Slamon DJ, Godolphin W, Jones LA, et al: Studies of the HER-2/neu proto-oncogene in human breast and ovarian cancer. Science 244: 707-712, 1989.

8. Andrulis IL, Bull SB, Blackstein ME, et al: neu/erbB-2 amplification identifies a poor-prognosis group of women with node-negative breast cancer. Toronto Breast Cancer Study Group. J Clin Oncol 16: 1340-1349, 1998.

9. Di Cristofano A and Pandolfi PP: The multiple roles of PTEN in tumor suppression. Cell 100: 387-390, 2000.
10. Hollander MC, Blumenthal GM and Dennis PA: PTEN loss in the continuum of common cancers, rare syndromes and mouse models. Nat Rev Cancer 11: 289-301, 2011.

11. Saal LH, Gruvberger-Saal SK, Persson C, et al: Recurrent gross mutations of the PTEN tumor suppressor gene in breast cancers with deficient DSB repair. Nat Genet 40: 102-107, 2008.

12. Esteva FJ, Guo H, Zhang S, et al: PTEN, PIK3CA, p-AKT, and p-p70S6K status: association with trastuzumab response and survival in patients with HER2-positive metastatic breast cancer. Am J Pathol 177: 1647-1656, 2010.

13. Gajria D and Chandarlapaty S: HER2-amplified breast cancer: mechanisms of trastuzumab resistance and novel targeted therapies. Expert Rev Anticancer Ther 11: 263-275, 2011.

14. Nagata Y, Lan KH, Zhou X, et al: PTEN activation contributes to tumor inhibition by trastuzumab, and loss of PTEN predicts trastuzumab resistance in patients. Cancer Cell 6: 117-127, 2004.

15. Schade B, Rao T, Dourdin N, et al: PTEN deficiency in a luminal ErbB-2 mouse model results in dramatic acceleration of mammary tumorigenesis and metastasis. J Biol Chem 284: 19018-19026, 2009.

16. Andrechek ER, Laing MA, Girgis-Gabardo AA, Siegel PM, Cardiff RD and Muller WJ: Gene expression profiling of neuinduced mammary tumors from transgenic mice reveals genetic and morphological similarities to ErbB2-expressing human breast cancers. Cancer Res 63: 4920-4926, 2003.

17. Wang $\mathrm{Q}$, Li SH, Wang $\mathrm{H}$, et al: Concomitant targeting of tumor cells and induction of T-cell response synergizes to effectively inhibit trastuzumab-resistant breast cancer. Cancer Res 72 : 4417-4428, 2012.

18. Andrechek ER, Hardy WR, Siegel PM, Rudnicki MA, Cardiff RD and Muller WJ: Amplification of the neu/erbB-2 oncogene in a mouse model of mammary tumorigenesis. Proc Natl Acad Sci USA 97: 3444-3449, 2000.

19. Ursini-Siegel J, Rajput AB, Lu H, et al: Elevated expression of DecR1 impairs ErbB2/Neu-induced mammary tumor development. Mol Cell Biol 27: 6361-6371, 2007.

20. Dourdin N, Schade B, Lesurf R, et al: Phosphatase and tensin homologue deleted on chromosome 10 deficiency accelerates tumor induction in a mouse model of ErbB-2 mammary tumorigenesis. Cancer Res 68: 2122-2131, 2008.

21. Wang Q, Ding H, Liu B, et al: Addition of the Akt inhibitor triciribine overcomes antibody resistance in cells from ErbB2/ Neu-positive/PTEN-deficient mammary tumors. Int J Oncol 44: 1277-1283, 2014

22. Frese KK and Tuveson DA: Maximizing mouse cancer models. Nat Rev Cancer 7: 645-658, 2007.

23. Vargo-Gogola T and Rosen JM: Modelling breast cancer: one size does not fit all. Nat Rev Cancer 7: 659-672, 2007.

24. Gopinathan A and Tuveson DA: The use of GEM models for experimental cancer therapeutics. Dis Model Mech 1: 83-86, 2008.

25. Sahin O, Wang Q, Brady SW, et al: Biomarker-guided sequential targeted therapies to overcome therapy resistance in rapidly evolving highly aggressive mammary tumors. Cell Res 24: 542-559, 2014.

26. Berns K, Horlings HM, Hennessy BT, et al: A functional genetic approach identifies the PI3K pathway as a major determinant of trastuzumab resistance in breast cancer. Cancer Cell 12:395-402, 2007.

27. Kruser TJ and Wheeler DL: Mechanisms of resistance to HER family targeting antibodies. Exp Cell Res 316: 1083-1100, 2010.

28. Hernandez-Aya LF and Gonzalez-Angulo AM: Targeting the phosphatidylinositol 3-kinase signaling pathway in breast cancer. Oncologist 16: 404-414, 2011.

29. Rosen LS, Ashurst HL and Chap L: Targeting signal transduction pathways in metastatic breast cancer: a comprehensive review. Oncologist 15: 216-235, 2010.

30. Ferris RL, Jaffee EM and Ferrone S: Tumor antigen-targeted, monoclonal antibody-based immunotherapy: clinical response, cellular immunity, and immunoescape. J Clin Oncol 28: 4390-4399, 2010.

31. Park S, Jiang Z, Mortenson ED, et al: The therapeutic effect of anti-HER2/neu antibody depends on both innate and adaptive immunity. Cancer Cell 18: 160-170, 2010.

32. Stagg J, Loi S, Divisekera U, et al: Anti-ErbB-2 mAb therapy requires type I and II interferons and synergizes with anti-PD-1 or anti-CD137 mAb therapy. Proc Natl Acad Sci USA 108: 7142-7147, 2011. 